\title{
AN ATOMIC DECOMPOSITION FOR HARDY SPACES ASSOCIATED TO SCHRÖDINGER OPERATORS
}

\author{
LIANG SONG, CHAOQIANG TAN ${ }^{\bowtie}$ and LIXIN YAN
}

(Received 2 August 2009; accepted 18 March 2011)

Communicated by A. M. Hassell

\begin{abstract}
Let $L=-\Delta+V$ be a Schrödinger operator on $\mathbb{R}^{n}$ where $V$ is a nonnegative function in the space $L_{\text {loc }}^{1}\left(\mathbb{R}^{n}\right)$ of locally integrable functions on $\mathbb{R}^{n}$. In this paper we provide an atomic decomposition for the Hardy space $H_{L}^{1}\left(\mathbb{R}^{n}\right)$ associated to $L$ in terms of the maximal function characterization. We then adapt our argument to give an atomic decomposition for the Hardy space $H_{L}^{1}\left(\mathbb{R}^{n} \times \mathbb{R}^{n}\right)$ on product domains.
\end{abstract}

2010 Mathematics subject classification: primary 42B30; secondary 42B25, 47F05.

Keywords and phrases: Hardy space, Schrödinger operator, atom, nontangential maximal function, product spaces.

\section{Introduction}

Let $V$ be a locally integrable nonnegative function on $\mathbb{R}^{n}$ (where $n \geq 1$ ), which is not identically zero. We define the form $Q$ by

$$
Q(u, v):=\int_{\mathbb{R}^{n}} \nabla u \nabla v d x+\int_{\mathbb{R}^{n}} V u v d x
$$

with domain

$$
\mathcal{D}(Q):=\left\{\left.u \in W^{1,2}\left(\mathbb{R}^{n}\right)\left|\int_{\mathbb{R}^{n}} V\right| u\right|^{2} d x<\infty\right\} .
$$

The space $W^{1,2}\left(\mathbb{R}^{n}\right)$ which appears in the formula above is the Sobolev space consisting of those $L^{2}$ functions on $\mathbb{R}^{n}$ whose gradients are also square integrable. It is well known that this symmetric form is closed. We recall that it was shown by Simon [25] that this form coincides with the minimal closure of the form given by the same expression but defined on $C_{0}^{\infty}\left(\mathbb{R}^{n}\right)$, the space of $C^{\infty}$ functions with compact support.

L. Song is supported by NNSF of China (No. 11001276 and 10926136) and NSF of Guangdong (No. 9451027501002491). C. Q. Tan is supported by NNSF of China (No. 11026215), NSF of Guangdong (No. 10451503101006384) and Specialized Research Fund for the Doctoral Program of Higher Education (No. 20104402120002). L. X. Yan is supported by NNSF of China (No. 10771221 and 10925106).

(c) 2011 Australian Mathematical Publishing Association Inc. 1446-7887/2011 \$16.00 
Let $L$ denote the self-adjoint operator associated with $Q$. The domain of $L$, written $\mathcal{D}(L)$, is defined to be the set of all $u \in \mathcal{D}(Q)$ for which there exists $v \in L^{2}$ such that

$$
Q(u, \varphi)=\int_{\mathbb{R}^{n}} v \bar{\varphi} d x \quad \forall \varphi \in \mathcal{D}(Q) .
$$

Formally, we write $L=-\Delta+V$ as a Schrödinger operator with potential $V$. Since $V$ is a locally integrable nonnegative function on $\mathbb{R}^{n}$, the Feynman-Kac formula implies that the kernel $p_{t}(x, y)$ of the semigroup $e^{-t L}$ satisfies the estimate

$$
0 \leq p_{t}(x, y) \leq(4 \pi t)^{-n / 2} e^{-|x-y|^{2} /(4 t)}
$$

for all $t>0$ and $x, y \in \mathbb{R}^{n}$ (see [24, p. 195]).

Given a function $f \in L^{2}\left(\mathbb{R}^{n}\right)$, we consider the following nontangential maximal function associated with the Poisson semigroup generated by the operator $L$ :

$$
f_{L}^{*}(x):=\sup _{|y-x|<t}\left|e^{-t \sqrt{L}} f(y)\right| \quad \forall x \in \mathbb{R}^{n} .
$$

The space $H_{L}^{1}\left(\mathbb{R}^{n}\right)$ is defined to be the completion of $L^{2}\left(\mathbb{R}^{n}\right)$ in the norm given by the $L^{1}$ norm of this maximal function, that is,

$$
\|f\|_{H_{L}^{1}\left(\mathbb{R}^{n}\right)}:=\left\|f_{L}^{*}\right\|_{L^{1}\left(\mathbb{R}^{n}\right)} .
$$

See, for example, $[1-3,12,17,18]$ for the properties of $H_{L}^{1}\left(\mathbb{R}^{n}\right)$.

Note that if $L=-\Delta$, then the space $H_{L}^{1}\left(\mathbb{R}^{n}\right)$ is the classical Hardy space $H^{1}\left(\mathbb{R}^{n}\right)$ which is a natural substitute for $L^{1}\left(\mathbb{R}^{n}\right)$. Recall that the development of the theory of the classical Hardy spaces in $\mathbb{R}^{n}$ was initiated by Stein and Weiss [26] and was originally tied closely to the theory of harmonic functions. Real variable methods were introduced into this subject in the seminal paper of Fefferman and Stein [15], the evolution of whose ideas led eventually to a characterization of Hardy spaces via the so-called 'atomic decomposition' obtained by Coifman [7] when $n=1$ and in higher dimensions by Latter [21].

An atomic decomposition for $H_{L}^{1}\left(\mathbb{R}^{n}\right)$ was given in [17] by combining the area $S$-function and the finite speed propagation property for the wave equation. Following [17], a function $a \in L^{2}\left(\mathbb{R}^{n}\right)$ is called a $(1,2)$-atom associated to the operator $L$ if there exists a function $b \in \mathcal{D}(L)$, the domain of an operator $L$ and a ball $B$ of $\mathbb{R}^{n}$ such that

$$
\begin{gathered}
a=L b ; \\
\operatorname{supp} L^{k} b \subseteq B ; \\
\left\|\left(r_{B}^{2} L\right)^{k} b\right\|_{L^{2}\left(\mathbb{R}^{n}\right)} \leq r_{B}^{2}|B|^{-1 / 2},
\end{gathered}
$$

where $k=0,1$ and $r_{B}$ denotes the radius of the ball $B$.

The aim of this paper is to get an atomic decomposition directly from the fact that $f_{L}^{*} \in L^{1}\left(\mathbb{R}^{n}\right)$ and then to provide a new proof of the atomic decomposition for $H_{L}^{1}\left(\mathbb{R}^{n}\right)$. Our first main result is the following theorem. 
THEOREM 1.1. Let $L=-\Delta+V$ where $V \in L_{\text {loc }}^{1}\left(\mathbb{R}^{n}\right)$ is a nonnegative function on $\mathbb{R}^{n}$. Let $f \in H_{L}^{1}\left(\mathbb{R}^{n}\right)$. Then there exist $(1,2)$-atoms $a_{j}$ and real numbers $\lambda_{j}$ for $j=1,2,3, \ldots$ such that

$$
f=\sum_{j=1}^{\infty} \lambda_{j} a_{j}
$$

in $H_{L}^{1}\left(\mathbb{R}^{n}\right)$. Furthermore, matters can be arranged so that the sequence $\lambda_{j}$ satisfies the inequality

$$
\sum_{j=1}^{\infty}\left|\lambda_{j}\right| \leq C\|f\|_{H_{L}^{1}\left(\mathbb{R}^{n}\right)}
$$

for some positive constant $C$, which may depend on $n$.

Conversely, any function $f$ which is written in the form of (1.2), where the $a_{j}$ are $(1,2)$-atoms, satisfies the inequality

$$
\|f\|_{H_{L}^{1}\left(\mathbb{R}^{n}\right)} \leq C \sum_{j=1}^{\infty}\left|\lambda_{j}\right| .
$$

We mention that the localized version of the atomic decomposition for $H_{L}^{1}\left(\mathbb{R}^{n}\right)$ when $L=-\Delta+V$ was given in [13], by using the properties of local Hardy spaces (see [16]), under the assumption that $V$ was a fixed nonnegative function on $\mathbb{R}^{n}$ belonging to the reverse Hölder class $B_{q}$ for some $q>1$. That is, there exists a positive constant $C$, possibly depending on $q$ and $V$, such that the reverse Hölder inequality

$$
\left(\frac{1}{|B|} \int_{B} V^{q} d x\right)^{1 / q} \leq C\left(\frac{1}{|B|} \int_{B} V d x\right)
$$

holds for every ball $B$ in $\mathbb{R}^{n}$.

Let us now turn to the Hardy space on product domains. We note that the usual space $H^{1}\left(\mathbb{R}^{n} \times \mathbb{R}^{n}\right)$ on the product domain is now well understood (see, for instance, $[4,5,14])$. In this paper we shall be concerned with the space $H_{L}^{1}\left(\mathbb{R}^{n} \times \mathbb{R}^{n}\right)$ associated to the Schrödinger operator $L$ (see [11] for more properties).

For any $\left(x_{1}, x_{2}\right) \in \mathbb{R}^{n} \times \mathbb{R}^{n}$ and $f \in L^{2}\left(\mathbb{R}^{n} \times \mathbb{R}^{n}\right)$, define

$$
f_{L}^{*}\left(x_{1}, x_{2}\right)=\sup _{\substack{\left|y_{1}-x_{1}\right|<t_{1} \\\left|y_{2}-x_{2}\right|<t_{2}}}\left|e^{-t_{1} \sqrt{L}} \otimes e^{-t_{2} \sqrt{L}} f\left(y_{1}, y_{2}\right)\right|
$$

where

$$
e^{-t_{1} \sqrt{L}} \otimes e^{-t_{2} \sqrt{L}} f\left(y_{1}, y_{2}\right):=\iint_{\mathbb{R}^{n} \times \mathbb{R}^{n}} p_{t_{1}}\left(y_{1}, z_{1}\right) p_{t_{2}}\left(y_{2}, z_{2}\right) f\left(z_{1}, z_{2}\right) d z_{1} d z_{2} .
$$

The space $H_{L}^{1}\left(\mathbb{R}^{n} \times \mathbb{R}^{n}\right)$ is defined to be the completion of $L^{2}\left(\mathbb{R}^{n} \times \mathbb{R}^{n}\right)$ in the norm given by

$$
\|f\|_{H_{L}^{1}\left(\mathbb{R}^{n} \times \mathbb{R}^{n}\right)}:=\left\|f_{L}^{*}\right\|_{L^{1}\left(\mathbb{R}^{n} \times \mathbb{R}^{n}\right)} .
$$

For our purposes, a product $(1,2)$-atom is a function $a$ on $\mathbb{R}^{2 n}$, together with an associated open set $\Omega$ of finite measure satisfying the following two properties. 
First, the function $a$ can be further decomposed into the form $a=\sum_{R \in m(\Omega)} a_{R}$ where for each $R \in m(\Omega)$ there exists a function $b_{R}$ such that $a_{R}=(L \otimes L) b_{R}$ and

$$
\operatorname{supp}\left(L^{i} \otimes L^{j}\right) b_{R} \subseteq 10 R, \quad i, j=0,1,
$$

where $10 R$ denotes the rectangle with the same center as $R$ and 10 times the side lengths.

Second,

$$
\|a\|_{L^{2}\left(\mathbb{R}^{n} \times \mathbb{R}^{n}\right)} \leq|\Omega|^{-1 / 2}
$$

and

$$
\sum_{R \in m(\Omega)} \sum_{i, j=0}^{1} \ell(I)^{4 i-4} \ell(J)^{4 j-4}\left\|\left(L^{i} \otimes L^{j}\right) b_{R}\right\|_{L^{2}\left(\mathbb{R}^{n} \times \mathbb{R}^{n}\right)}^{2} \leq|\Omega|^{-1}
$$

where $R=I \times J$ denotes the dyadic rectangle of $\mathbb{R}^{n} \times \mathbb{R}^{n}$ whose side lengths are $\ell(I)$ and $\ell(J), 10 R$ denotes the set $\{10 x \mid x \in R\}$ and $m(\Omega)$ denotes the set of maximal dyadic subrectangles of $\Omega$ (see Section 4 below).

The second main result of this paper is the following theorem.

THEOREM 1.2. Let $L=-\Delta+V$ where $V \in L_{\text {loc }}^{1}\left(\mathbb{R}^{n}\right)$ is a nonnegative function on $\mathbb{R}^{n}$. Let $f \in H_{L}^{1}\left(\mathbb{R}^{n} \times \mathbb{R}^{n}\right)$. Then there exist product $(1,2)$-atoms $a_{j}$ and numbers $\lambda_{j}$, where $j=0,1,2, \ldots$, such that

$$
f=\sum_{j=1}^{\infty} \lambda_{j} a_{j}
$$

in $H_{L}^{1}\left(\mathbb{R}^{n} \times \mathbb{R}^{n}\right)$, and the sequence $\lambda_{j}$ satisfies the condition that

$$
\sum_{j=1}^{\infty}\left|\lambda_{j}\right| \leq C\|f\|_{H_{L}^{1}\left(\mathbb{R}^{n} \times \mathbb{R}^{n}\right)}
$$

Conversely, for any decomposition of $f$ of the form in (1.3),

$$
\|f\|_{H_{L}^{1}\left(\mathbb{R}^{n} \times \mathbb{R}^{n}\right)} \leq C \sum_{j=1}^{\infty}\left|\lambda_{j}\right|
$$

The organisation of this paper is as follows. In Section 2 we introduce some notation and preliminary lemmas. Our main results, Theorems 1.1 and 1.2, are proved in Sections 3 and 4. The main contribution of this paper is to combine the Calderón reproducing formula, the finite propagation speed property and the methods of Wilson [27] to obtain an atomic decomposition of Hardy spaces and then to verify the required $L^{2}$ norm estimates of the atoms by using square function estimates.

Throughout this paper, the letters $C$ and $c$ denote (possibly different) constants that are independent of the essential variables.

\section{Preliminaries}

Recall that if $L$ is a nonnegative, self-adjoint operator on $L^{2}\left(\mathbb{R}^{n}\right)$ and $E_{L}(\lambda)$ denotes a spectral decomposition associated with $L$, then for every bounded Borel 
function $F:[0, \infty) \rightarrow \mathbb{C}$ one defines the operator

$$
F(L): L^{2}\left(\mathbb{R}^{n}\right) \rightarrow L^{2}\left(\mathbb{R}^{n}\right)
$$

by the formula

$$
F(L):=\int_{0}^{\infty} F(\lambda) d E_{L}(\lambda) .
$$

In particular, the operator $\cos (t \sqrt{L})$ is well defined on $L^{2}\left(\mathbb{R}^{n}\right)$. Moreover, it follows from [9, Theorem 3] (see also [6]) that the integral kernel $K_{\cos (t \sqrt{L})}$ of $\cos (t \sqrt{L})$ satisfies

$$
\operatorname{supp} K_{\cos (t \sqrt{L})} \subseteq\left\{(x, y) \in \mathbb{R}^{n} \times \mathbb{R}^{n}|| x-y \mid \leq t\right\} .
$$

By the Fourier inversion formula, whenever $F$ is an even bounded Borel function with Fourier transform $\hat{F}$ in $L^{1}(\mathbb{R})$, we can write $F(\sqrt{L})$ in terms of $\cos (t \sqrt{L})$. Specifically, using (2.1), we have

$$
F(\sqrt{L})=(2 \pi)^{-1} \int_{-\infty}^{\infty} \hat{F}(t) \cos (t \sqrt{L}) d t
$$

which, when combined with (2.2), gives us that

$$
K_{F(\sqrt{L})}=(2 \pi)^{-1} \int_{|t| \geq|x-y|} \hat{F}(t) K_{\cos (t \sqrt{L})} d t .
$$

Lemma 2.1. Let $\varphi \in C_{0}^{\infty}(\mathbb{R})$ be an even function such that $\operatorname{supp} \varphi \subseteq[-1,1]$. Let $\Phi$ denote the Fourier transform of $\varphi$. Then for every $\kappa=0,1,2, \ldots$ and for every $t>0$ the kernel $K_{\left(t^{2} L\right)^{\kappa} \Phi(t \sqrt{L})}$ of $\left(t^{2} L\right)^{\kappa} \Phi(t \sqrt{L})$ satisfies the condition

$$
\operatorname{supp} K_{\left(t^{2} L\right)^{\kappa} \Phi(t \sqrt{L})} \subseteq\left\{(x, y) \in \mathbb{R}^{n} \times \mathbb{R}^{n}|| x-y \mid \leq t\right\} .
$$

Proof. We refer the reader to [17, Lemma 3.5] for the proof.

In this paper we use $\mathbb{R}_{+}^{n+1}$ to denote the upper half space of $\mathbb{R}^{n+1}$. In the following lemma we shall assume that $\varphi \in C_{0}^{1}\left(\mathbb{R}^{n}\right)$ is nonnegative, radial and nonincreasing. We also assume that $\varphi=1$ on $B(0,1 / 2)$, supp $\varphi \subseteq B(0,1)$ and $\int \varphi(x) d x=1$. We sometimes use capital letters to denote points of $\mathbb{R}_{+}^{n+1}$ (for example, $X=(x, t)$ ), and set

$$
\begin{gathered}
u(x, t)=e^{-t \sqrt{L}} f(x), \\
\nabla_{X} u(X)=\left(\nabla_{x} u, \partial_{t} u\right) \\
\left|\nabla_{X} u\right|^{2}=\left|\nabla_{x} u\right|^{2}+\left|\partial_{t} u\right|^{2} .
\end{gathered}
$$

Lemma 2.2. For every $f, g \in L^{2}\left(\mathbb{R}^{n}\right)$,

$$
\begin{aligned}
& \iint_{\mathbb{R}_{+}^{n+1}}\left|t \nabla_{X} u(x, t)\right|^{2}\left|\varphi_{t} * g(x)\right|^{2} \frac{d x d t}{t} \\
& \quad \leq \int_{\mathbb{R}^{n}}|f(x)|^{2}|g(x)|^{2} d x+\iint_{\mathbb{R}_{+}^{n+1}}|u(x, t)|^{2}\left|\psi_{t} * g(x)\right|^{2} \frac{d x d t}{t}
\end{aligned}
$$

where $\psi$ is a vector-valued function with the same support as $\varphi$ and mean value 0. 
Proof. The proof of Lemma 2.2 can be obtained by making minor modifications to the proof of [23, Lemma 3.1] in the case where $L=-\Delta$ is the Laplace operator on $\mathbb{R}^{n}$. For the sake of completeness and for the reader's convenience we give a brief sketch of the proof of this lemma.

Write $\nabla_{X}^{2}=\nabla_{X} \nabla_{X}$. Since $u=e^{-t \sqrt{L}} f$, we have

$$
\nabla_{X}^{2} u^{2}=\left(\partial_{t}^{2}+\Delta\right) u^{2}=2\left|\nabla_{X} u\right|^{2}+2 V u^{2} .
$$

This, together with the condition that $V \geq 0$, gives

$$
\begin{aligned}
& 2 \iint_{\mathbb{R}_{+}^{n+1}}\left|t \nabla_{X} u\right|^{2}\left|\varphi_{t} * g\right|^{2} \frac{d x d t}{t} \\
& \quad=\iint_{\mathbb{R}_{+}^{n+1}} \nabla_{X}^{2} u^{2}\left|\varphi_{t} * g\right|^{2} t d x d t-2 \iint_{\mathbb{R}_{+}^{n+1}} V u^{2}\left|\varphi_{t} * g\right|^{2} t d x d t \\
& \quad \leq \iint_{\mathbb{R}_{+}^{n+1}} \nabla_{X}^{2} u^{2}\left|\varphi_{t} * g\right|^{2} t d x d t .
\end{aligned}
$$

After an integration by parts we obtain

$$
\begin{aligned}
\iint_{\mathbb{R}_{+}^{n+1}} & \nabla_{X}^{2} u^{2}\left|\varphi_{t} * g\right|^{2} t d x d t \\
= & -\iint_{\mathbb{R}_{+}^{n+1}} \nabla_{X} u^{2} \nabla_{X}\left(t\left(\varphi_{t} * g\right)^{2}\right) d x d t \\
= & -2 \iint_{\mathbb{R}_{+}^{n+1}}\left(2 u \nabla_{X} u\left(\varphi_{t} * g\right) t \nabla_{X}\left(\varphi_{t} * g\right)+u \partial_{t} u\left|\varphi_{t} * g\right|^{2}\right) d x d t .
\end{aligned}
$$

Note that the conditions $u(x, 0) \in L^{2}\left(\mathbb{R}^{n}\right)$ or $f \in L^{2}\left(\mathbb{R}^{n}\right)$ are sufficient to ensure that the boundary terms 'at $\infty$ ' for this integration by parts vanish, as does the boundary term for $t=0$.

We use a further integration by parts to obtain

$$
\begin{aligned}
& 2 \iint_{\mathbb{R}_{+}^{n+1}} u \partial_{t} u\left(\varphi_{t} * g\right)^{2} d x d t \\
& \quad=-\lim _{t \rightarrow 0} \int_{\mathbb{R}}^{n} u^{2}\left(\varphi_{t} * g\right)^{2} d x d t-2 \iint_{\mathbb{R}_{+}^{n+1}} u^{2}\left(\varphi_{t} * g\right)\left(\partial_{t}\left(\varphi_{t} * g\right)\right) d x d t \\
& \quad=-\int_{\mathbb{R}}^{n} f^{2} g^{2} d x-2 \iint_{\mathbb{R}_{+}^{n+1}} u^{2}\left(\varphi_{t} * g\right)\left(\partial_{t}\left(\varphi_{t} * g\right)\right) d x d t
\end{aligned}
$$

When combined with (2.4), integration by parts and the Cauchy-Schwarz inequality, this gives (2.3) provided that

$$
\left.\left|\psi_{t} * f\right|^{2}=9 \mid\left(t \nabla_{x} \varphi_{t}\right) * g\right)\left.\right|^{2}+9\left|\vec{\rho}_{t} * g\right|^{2} .
$$

Here $\vec{\rho}=\left(x_{1} \varphi, \ldots, x_{n} \varphi\right)$. For the details, we refer the reader to [23, (3.8)]. This completes our proof. 
Finally for $s>0$ we define the set of measurable functions

$$
\mathbb{F}(s):=\left\{\psi: \mathbb{C} \rightarrow \mathbb{C}|| \psi(z) \mid \leq C \frac{|z|^{s}}{\left(1+|z|^{2 s}\right)}\right\} .
$$

Then for any nonzero function $\psi \in \mathbb{F}(s)$,

$$
\left\{\int_{0}^{\infty}|\psi(t)|^{2} d t / t\right\}^{1 / 2}<\infty .
$$

We write $\psi_{t}(z)=\psi(t z)$. It follows from spectral theory (see [10]) that, if $f \in L^{2}\left(\mathbb{R}^{n}\right)$, then

$$
\begin{aligned}
\left\{\int_{0}^{\infty}\|\psi(t \sqrt{L}) f\|_{L^{2}\left(\mathbb{R}^{n}\right)}^{2} \frac{d t}{t}\right\}^{1 / 2} & =\left\{\int_{0}^{\infty}\langle\bar{\psi}(t \sqrt{L}) \psi(t \sqrt{L}) f, f\rangle \frac{d t}{t}\right\}^{1 / 2} \\
& =\left\{\left\langle\int_{0}^{\infty}|\psi|^{2}(t \sqrt{L}) \frac{d t}{t} f, f\right\rangle\right\}^{1 / 2} \\
& =\kappa\|f\|_{L^{2}\left(\mathbb{R}^{n}\right)}
\end{aligned}
$$

where $\kappa=\left\{\int_{0}^{\infty}|\psi(t)|^{2} d t / t\right\}^{1 / 2}$.

\section{Proof of Theorem 1.1}

We shall use $\mathcal{M}$ to denote the Hardy-Littlewood maximal function with respect to the balls of $\mathbb{R}^{n}$. We use the notation

$$
\Gamma(x)=\left\{(y, t) \in \mathbb{R}_{+}^{n+1}|| x-y \mid<t\right\}
$$

to denote the standard cone (of aperture 1) with vertex $x \in \mathbb{R}^{n}$.

For any closed subset $F$ of $\mathbb{R}^{n}$, we denote by $\mathcal{R}(F)$ the union of all cones with vertices in $F$, that is,

$$
\mathcal{R}(F)=\bigcup_{x \in F} \Gamma(x) .
$$

If $O$ is an open subset of $\mathbb{R}^{n}$, then the 'tent' over $O$, denoted by $\widehat{O}$, is defined to be

$$
\widehat{O}={ }^{c}\left[\mathcal{R}\left({ }^{c} O\right)\right] .
$$

Proof of Theorem 1.1. Let $f \in H_{L}^{1}\left(\mathbb{R}^{n}\right) \cap L^{2}\left(\mathbb{R}^{n}\right)$. We shall prove that $f$ has an atomic decomposition as in (1.2). We start with a suitable version of the Calderón reproducing formula.

Let $\varphi$ and $\Phi$ be as in Lemma 2.1 and set $\Psi(x):=x^{4} \Phi(x)$ for all $x \in \mathbb{R}$. By the $L^{2}$ functional calculus (see [22]) for every $f \in L^{2}\left(\mathbb{R}^{n}\right)$ we can write

$$
\begin{aligned}
f & =c_{\Psi} \int_{0}^{\infty} \Psi(t \sqrt{L}) t \sqrt{L} e^{-t \sqrt{L}} f \frac{d t}{t} \\
& =\lim _{N \rightarrow \infty} c_{\Psi} \int_{1 / N}^{N} \Psi(t \sqrt{L}) t \sqrt{L} e^{-t \sqrt{L}} f \frac{d t}{t}
\end{aligned}
$$

with the integral converging in $L^{2}\left(\mathbb{R}^{n}\right)$. 
For $i \in \mathbb{Z}$ we define the sets

$$
O_{i}:=\left\{x \in \mathbb{R}^{n} \mid f_{L}^{*}(x)>2^{i}\right\}
$$

and consider

$$
O_{i}^{*}=\left\{x \in \mathbb{R}^{n} \mid \mathcal{M}\left(\chi_{O_{i}}\right)(x)>2^{-(n+1)}\right\} .
$$

Then $O_{i} \subseteq O_{i}^{*}$ and $\left|O_{i}^{*}\right| \leq C\left|O_{i}\right|$ for every $i \in \mathbb{Z}$.

Now let $\left\{Q_{i}^{j}\right\}_{j}$ be a Whitney decomposition of $O_{i}^{*}$ and let $\widehat{O_{i}^{*}}$ be a tent region, that is,

$$
\widehat{O_{i}^{*}}:=\left\{(x, t) \in \mathbb{R}^{n} \times(0, \infty) \mid \operatorname{dist}\left(x,{ }^{c} O_{i}^{*}\right) \geq t\right\} .
$$

For every $i, j \in \mathbb{Z}$ we define

$$
T_{i}^{j}=\left(Q_{i}^{j} \times(0,+\infty)\right) \cap \widehat{O_{i}^{*}} \backslash \widehat{O_{i+1}^{*}},
$$

and $\lambda_{i}^{j}=2^{i}\left|Q_{i}^{j}\right|$. Using formula (3.1), we write

$$
\begin{aligned}
f & =\sum_{j, i \in \mathbb{Z}} c_{\Psi} \int_{0}^{\infty} \Psi(t \sqrt{L})\left(\chi_{T_{i}^{j}} t \sqrt{L} e^{-t \sqrt{L}}\right) f \frac{d t}{t} \\
& =: \sum_{j, i \in \mathbb{Z}} \lambda_{i}^{j} a_{i}^{j}
\end{aligned}
$$

where $a_{i}^{j}=L b_{i}^{j}$ and

$$
b_{i}^{j}=\left(\lambda_{i}^{j}\right)^{-1} c_{\Psi} \int_{0}^{\infty} t^{4} L \Phi(t \sqrt{L})\left(\chi_{T_{i}^{j}} t \sqrt{L} e^{-t \sqrt{L}}\right) f \frac{d t}{t} .
$$

We claim that, up to normalization by a multiplicative constant, the $a_{i}^{j}$ are $(1,2)$-atoms. Once this claim is established, we shall have

$$
\begin{aligned}
\sum_{j, i}\left|\lambda_{i}^{j}\right| & =\sum_{j, i} 2^{i}\left|Q_{i}^{j}\right| \leq C \sum_{i} 2^{i}\left|O_{i}^{*}\right| \\
& \leq C \sum_{i} 2^{i}\left|O_{i}\right| \leq C\|f\|_{H_{L}^{1}\left(\mathbb{R}^{n}\right)}
\end{aligned}
$$

as desired.

Let us now prove the claim. We shall show that for every $j, i \in \mathbb{Z}$, the function $C^{-1} a_{i}^{j}$ is a $(1,2)$-atom associated with the cube $10 \sqrt{n} Q_{i}^{j}$ for some constant $C$ (independent of $i$ and $j)$. Observe that if $(x, t) \in T_{i}^{j}$, then $B(x, t) \in O_{i}^{*}$. This, together with the fact that $Q_{i}^{j}$ is the Whitney cube of $O_{i}^{*}$, allows us to deduce that

$$
t \leq 6 \sqrt{n} \ell\left(Q_{i}^{j}\right)
$$

By Lemma 2.1 the integral kernel $K_{\Phi_{t}(\sqrt{L})}$ of the operator $\Phi_{t}(\sqrt{L})$ satisfies the condition that

$$
\operatorname{supp} K_{\Phi_{t}(\sqrt{L})} \subseteq\left\{(x, y) \in \mathbb{R}^{n} \times \mathbb{R}^{n}|| x-y \mid \leq t\right\} .
$$


This enables us to deduce that, whenever $k=0,1$,

$$
\operatorname{supp}\left(L^{k} b_{i}^{j}\right) \subseteq 10 \sqrt{n} Q_{i}^{j} .
$$

To continue, for each cube $Q_{i}^{j}$ we consider some $h \in L^{2}\left(Q_{i}^{j}\right)$ such that $\|h\|_{L^{2}\left(Q_{i}^{j}\right)}=1$. Then for $k=0,1$,

$$
\begin{aligned}
\left|\lambda_{i}^{j} \int_{\mathbb{R}^{n}}\left(\ell\left(Q_{i}^{j}\right)^{2} L\right)^{k} b_{i}^{j}(x) h(x) d x\right| & \\
= & c \Psi\left|\int_{\mathbb{R}_{+}^{n+1}} t^{4}\left(\ell\left(Q_{i}^{j}\right)^{2} L\right)^{k} L \Phi(t \sqrt{L})\left(\chi_{T_{i}^{j}} t \sqrt{L} e^{-t \sqrt{L}}\right) f(x) h(x) \frac{d x d t}{t}\right| \\
\leq & C \ell\left(Q_{i}^{j}\right)^{2} \int_{\mathbb{R}_{+}^{n+1}}\left|\left(\chi_{T_{i}^{j}} t \sqrt{L} e^{-t \sqrt{L}}\right) f(x) t^{2(k+1)} L^{k+1} \Phi(t \sqrt{L})(h)(x)\right| \frac{d x d t}{t} \\
\leq & C \ell\left(Q_{i}^{j}\right)^{2}\left(\iint_{T_{i}^{j}}\left|t \sqrt{L} e^{-t \sqrt{L}} f(x)\right|^{2} \frac{d x d t}{t}\right)^{1 / 2} \\
& \times\left(\iint_{\mathbb{R}_{+}^{n+1}}\left|\left(t^{2} L\right)^{k+1} \Phi(t \sqrt{L})(h)(x)\right|^{2} \frac{d x d t}{t}\right)^{1 / 2} \\
\leq & C \ell\left(Q_{i}^{j}\right)^{2}\left(\iint_{T_{i}^{j}}\left|t \sqrt{L} e^{-t \sqrt{L}} f(x)\right|^{2} \frac{d x d t}{t}\right)^{1 / 2} \cdot
\end{aligned}
$$

Note that the first inequality is obtained from the fact that $0<t<6 \sqrt{n} \ell\left(Q_{i}^{j}\right)$ and the third inequality follows from (2.5).

Therefore, in order to prove our claim, it suffices to show that

$$
\int_{T_{i}^{j}}\left|t \sqrt{L} e^{-t \sqrt{L}} f(y)\right|^{2} \frac{d y d t}{t} \leq C 2^{2 i}\left|Q_{i}^{j}\right| .
$$

Let us show that (3.2) is satisfied. Let $\varphi \in C_{0}^{\infty}\left(\mathbb{R}^{n}\right)$ be as in Lemma 2.2 and set

$$
F_{i}^{j}=10 \sqrt{n} Q_{i}^{j} \backslash O_{i+1} .
$$

We first show that, for all $(y, t) \in T_{i}^{j}$,

$$
\left|\varphi_{t} * \chi_{F_{i}^{j}}(y)\right| \geq C
$$

Indeed, for any $(y, t) \in T_{i}^{j}$, we can obtain

$$
B(y, t) \subseteq 10 \sqrt{n} Q_{i}^{j}
$$

and

$$
B(y, t) \cap{ }^{c} O_{i+1}^{*} \neq \varnothing .
$$

This shows that there exists $x_{0} \in B(y, t) \cap{ }^{c} O_{i+1}^{*}$ such that

$$
\mathcal{M}\left(\chi_{O_{i+1}}\right)\left(x_{0}\right) \leq 2^{-(n+1)} \text {. }
$$

It then follows that

$$
\left|B(y, t) \cap O_{i+1}\right| \leq 2^{-(n+1)}|B(y, t)| .
$$


This implies that

$$
\begin{aligned}
\left|B(y, t / 2) \cap F_{i}^{j}\right| & \geq\left|B(y, t / 2) \cap 10 \sqrt{n} Q_{i}^{j}\right|-\left|B(y, t / 2) \cap O_{i+1}\right| \\
& \geq|B(y, t / 2)|-2^{-(n+1)}|B(y, t)| \\
& =2^{-(n+1)}|B(y, t)|
\end{aligned}
$$

and then, for any $(y, t) \in T_{i}^{j}$,

$$
\left|\varphi_{t} * \chi_{F_{i}^{j}}(y)\right|=\left|\int \varphi_{t}(y-z) \chi_{F_{i}^{j}}(z) d z\right| \geq t^{-n}\left|B(y, t / 2) \cap F_{k}^{j}\right| \geq C
$$

which proves estimate (3.3).

By Lemma 2.2, we have

$$
\begin{aligned}
\int_{T_{i}^{j}} \mid t & \left.\sqrt{L} e^{-t \sqrt{L}} f(y)\right|^{2} \frac{d y d t}{t} \\
\leq & C \int_{\mathbb{R}_{+}^{n+1}}\left|t \nabla_{X} e^{-t \sqrt{L}} f(y)\right|^{2}\left|\varphi_{t} * \chi_{F_{i}^{j}}(y)\right|^{2} \frac{d y d t}{t} \\
\leq & C\left(\int_{\mathbb{R}_{+}^{n+1}}\left|e^{-t \sqrt{L}} f(y)\right|^{2}\left|\psi_{t} * \chi_{F_{i}^{j}}(y)\right|^{2} \frac{d y d t}{t}+\int_{\mathbb{R}^{n}}|f(x)|^{2}\left|\chi_{F_{i}^{j}}(x)\right|^{2} d x\right) \\
& =: \mathrm{T}_{1}+\mathrm{T}_{2} .
\end{aligned}
$$

Observe that if $\psi_{t} * \chi_{F_{i}^{j}}(y) \neq 0$, then $F_{i}^{j} \cap B(y, t) \neq \varnothing$ and there exists an element

$$
x_{0} \in B(y, t) \cap\left(10 \sqrt{n} Q_{i}^{j}\right) \cap{ }^{c} O_{i+1} .
$$

This gives us that

$$
\left|e^{-t \sqrt{L}} f(y)\right| \leq f_{L}^{*}\left(x_{0}\right) \leq 2^{i+1}
$$

Hence

$$
\mathrm{T}_{1} \leq C 2^{2 i+2} \int_{\mathbb{R}_{+}^{n+1}}\left|\psi_{t} * \chi_{F_{i}^{j}}(y)\right|^{2} \frac{d y d t}{t} \leq C 2^{2 i}\left|Q_{i}^{j}\right| .
$$

Also

$$
\mathrm{T}_{2} \leq C 2^{2 i+2} \int_{\mathbb{R}^{n}}\left|\chi_{F_{i}^{j}}(x)\right|^{2} d x \leq C 2^{2 i}\left|Q_{i}^{j}\right|
$$

and the estimate (3.2) follows readily.

We have shown that, up to normalization by a multiplicative constant, the $a_{i}^{j}$ are $(1,2)$-atoms associated with the ball $B\left(x_{i}^{j}, c_{1} \ell\left(Q_{i}^{j}\right)\right)$ for some constant $c_{1}$ where $x_{i}^{j}$ is the center of the cube $Q_{i}^{j}$. This proves that $f$ has an atomic decomposition as in (1.2).

To prove the converse we assume that $f=\sum_{j} \lambda_{j} a_{j}$ where the $a_{j}$ are $(1,2)$-atoms and $\sum_{j}\left|\lambda_{j}\right|<\infty$. In this case, it was proved in [17, Theorem 7.4] that $f \in H_{L}^{1}\left(\mathbb{R}^{n}\right)$. We omit the details here. The proof of Theorem 1.1 is now complete.

\section{Proof of Theorem $\mathbf{1 . 2}$}

In this section we shall work exclusively with the domain $\mathbb{R}_{+}^{n+1} \times \mathbb{R}_{+}^{n+1}$ and its distinguished boundary $\mathbb{R}^{n} \times \mathbb{R}^{n}$. If $x=\left(x_{1}, x_{2}\right) \in \mathbb{R}^{n} \times \mathbb{R}^{n}$, then we shall denote 
by $\Gamma(x)$ the product cone $\Gamma(x)=\Gamma\left(x_{1}\right) \times \Gamma\left(x_{2}\right)$, where

$$
\Gamma\left(x_{i}\right)=\left\{\left(y_{i}, t_{i}\right) \in \mathbb{R}_{+}^{n+1}|| x_{i}-y_{i} \mid<t_{i}\right\}
$$

for $i=1,2$. If $(x, t):=\left(\left(x_{1}, t_{1}\right),\left(x_{2}, t_{2}\right)\right) \in \mathbb{R}_{+}^{n+1} \times \mathbb{R}_{+}^{n+1}$, then we shall write

$$
B_{x, t}:=B\left(x_{1}, t_{1}\right) \times B\left(x_{2}, t_{2}\right)
$$

for the product ball.

For any open set $\Omega \subseteq \mathbb{R}^{2 n}$, the tent over $\Omega$, denoted by $\hat{\Omega}$, is the set

$$
\left\{(x, t) \in \mathbb{R}_{+}^{n+1} \times \mathbb{R}_{+}^{n+1} \mid B_{x, t} \subseteq \Omega\right\} .
$$

Let $m(\Omega)$ denote the set of maximal dyadic subrectangles of $\Omega$. Let $m_{1}(\Omega)$ denote the subset of those dyadic subrectangles $R=I \times J$ of $\Omega$ that are maximal in the $x_{1}$ direction. In other words, if $S=I^{\prime} \times J \supseteq R$ is a dyadic subrectangle of $\Omega$, then $I=I^{\prime}$. Similarly, define $m_{2}(\Omega)$ to be the collection of those dyadic subrectangles of $\Omega$ that are maximal in the $x_{2}$ direction. Let $\mathcal{M}_{s}$ denote the strong maximal operator, that is, for any $x \in \mathbb{R}^{2 n}$ let

$$
\mathcal{M}_{s} f(x)=\sup _{t_{1}>0, t_{2}>0} \frac{1}{\left|B_{x, t}\right|} \int_{B_{x, t}}\left|f\left(y_{1}, y_{2}\right)\right| d y_{1} d y_{2} .
$$

In order to prove Theorem 1.2 we need some auxiliary results. The first one is Journé's covering lemma (see [20]).

Lemma 4.1. Let $\Omega$ be an open subset of $\mathbb{R}^{n} \times \mathbb{R}^{n}$ and let $R=I \times J \in m_{2}(\Omega)$ where $I, J$ are dyadic cubes of $\mathbb{R}^{n}$. Suppose that $\hat{I}$ is the biggest dyadic cube of $\mathbb{R}^{n}$ containing $I$ such that $\hat{I} \times J \subseteq \Omega^{*}$ where

$$
\Omega^{*}=\left\{x \in \mathbb{R}^{2 n} \mid \mathcal{M} \chi_{\Omega}(x)>1 / 2\right\} .
$$

We set $\gamma_{1}(R)=|\hat{I}| /|I|$ and define $\gamma_{2}$ similarly. Then for any $\delta>0$,

$$
\sum_{R \in m_{2}(\Omega)}|R| \gamma_{1}^{-\delta}(R) \leq c_{\delta}|\Omega|
$$

and

$$
\sum_{R \in m_{1}(\Omega)}|R| \gamma_{2}^{-\delta}(R) \leq c_{\delta}|\Omega|
$$

where $c_{\delta}$ is a constant depending only on $\delta$ and not on $\Omega$.

For every $i=1,2$ we let $\nabla_{X_{i}}=\left(\nabla_{x_{i}}, \partial_{t_{i}}\right)$. In the following lemma we assume that $\varphi \in C_{0}^{1}\left(\mathbb{R}^{n}\right)$ is nonnegative, radial and nonincreasing. We also assume that $\varphi=1$ on $B(0,1 / 2)$, supp $\varphi \subseteq B(0,1)$ and $\int \varphi(x) d x=1$.

Lemma 4.2. For every $f, g \in L^{2}\left(\mathbb{R}^{2 n}\right)$ and $i=1,2$ there exist vector-valued functions $\psi^{(i)} \in C_{0}^{\infty}\left(\mathbb{R}^{n}\right)$ satisfying the conditions $\operatorname{supp} \psi^{(i)} \subseteq B(0,1), \quad \int_{\mathbb{R}^{n}} \psi^{(i)}(x) d x=0$ and 
such that

$$
\begin{aligned}
\int_{\mathbb{R}_{+}^{n+1} \times \mathbb{R}_{+}^{n+1}}\left|t_{1} \nabla_{X_{1}} e^{-t_{1} \sqrt{L}} \otimes t_{2} \nabla_{X_{2}} e^{-t_{2} \sqrt{L}} f\left(y_{1}, y_{2}\right)\right|^{2}\left|\left(\varphi_{t_{1}} \otimes \varphi_{t_{2}}\right) * g\left(y_{1}, y_{2}\right)\right|^{2} \frac{d y d t}{t_{1} t_{2}} \\
\leq \int_{\mathbb{R}_{+}^{n+1} \times \mathbb{R}_{+}^{n+1}}\left|e^{-t_{1} \sqrt{L}} \otimes e^{-t_{2} \sqrt{L}} f\left(y_{1}, y_{2}\right)\right|^{2}\left|\left(\psi_{t_{1}}^{(1)} \otimes \psi_{t_{2}}^{(2)}\right) * g\left(y_{1}, y_{2}\right)\right|^{2} \frac{d y d t}{t_{1} t_{2}} \\
\quad+\int_{\mathbb{R}_{+}^{n+1} \times \mathbb{R}^{n}}\left|e^{-t_{1} \sqrt{L}} f\left(y_{1}, x_{2}\right)\right|^{2}\left|\psi_{t_{1}}^{(1)} * g\left(y_{1}, x_{2}\right)\right|^{2} \frac{d y_{1} d t_{1}}{t_{1}} d x_{2} \\
\quad+\int_{\mathbb{R}^{n} \times \mathbb{R}_{+}^{n+1}}\left|e^{-t_{2} \sqrt{L}} f\left(x_{1}, y_{2}\right)\right|^{2}\left|\psi_{t_{2}}^{(2)} * g\left(x_{1}, y_{2}\right)\right|^{2} \frac{d y_{2} d t_{2}}{t_{2}} d x_{1} \\
\quad+\int_{\mathbb{R}^{n} \times \mathbb{R}^{n}}\left|f\left(x_{1}, x_{2}\right)\right|^{2}\left|g\left(x_{1}, x_{2}\right)\right|^{2} d x_{1} d x_{2} .
\end{aligned}
$$

Proof. Repeated applications of Lemma 2.2 can be used to prove Lemma 4.2.

Finally we state the following lemma whose proof we omit since it is similar to that of [17, Lemma 4.3].

LEMma 4.3. Suppose that $T$ is a bounded sublinear operator on $L^{2}\left(\mathbb{R}^{2 n}\right)$ and that for every product $(1,2)$-atom a on product domains we have

$$
\|T a\|_{L^{1}\left(\mathbb{R}^{2 n}\right)} \leq C
$$

where the constant $C$ is independent of $a$. Then for any decomposition of the form given in (1.3) of $f$ we have

$$
\|T f\|_{L^{1}\left(\mathbb{R}^{2 n}\right)} \leq C \sum_{j=1}^{\infty}\left|\lambda_{j}\right| .
$$

Proof of Theorem 1.2 By condition (1.1) for every $K=0,1, \ldots$ there exists a constant $C_{K}$ such that the kernel $p_{t, K}$ of the operator $(t \sqrt{L})^{2 K} e^{-t \sqrt{L}}$ satisfies the condition that

$$
\left|p_{t, K}(x, y)\right| \leq C_{K} \frac{t}{(t+|x-y|)^{n+1}} \quad \forall t>0
$$

and almost every $x, y \in \mathbb{R}^{n}$ (see, for instance, [17, Lemma 7.2]).

Step 1. Let $f=\sum_{j} \lambda_{j} a_{j}$ where the $a_{j}$ are product $(1,2)$-atoms and $\sum_{j=1}^{\infty}\left|\lambda_{j}\right|<\infty$. Recall that the strong maximal operator $\mathcal{M}_{s}$ defined in (4.1) is bounded on $L^{2}\left(\mathbb{R}^{2 n}\right)$ (see [14]). This, together with condition (1.1), gives us that

$$
\left\|f_{L}^{*}\right\|_{L^{2}\left(\mathbb{R}^{2 n}\right)} \leq C\left\|\mathcal{M}_{S} f\right\|_{L^{2}\left(\mathbb{R}^{2 n}\right)} \leq C\|f\|_{L^{2}\left(\mathbb{R}^{2 n}\right)} .
$$

By Lemma 4.3, it is enough to show that $\left\|a_{L}^{*}\right\|_{L^{1}\left(\mathbb{R}^{2 n}\right)} \leq C$ for every product $(1,2)$-atom $a$, for some constant $C$ which is independent of $a$. 
Suppose that

$$
a=\sum_{R \in m(\Omega)} a_{R}=\sum_{R \in m(\Omega)}(L \otimes L) b_{R}
$$

is a product $(1,2)$-atom supported on some open subset $\Omega$ of $\mathbb{R}^{2 n}$. For any maximal dyadic subrectangle $R=I \times J \in m(\Omega)$ let $\ell(I), \ell(J)$ be the side-lengths of cubes $I$ and $J$ and let $I^{\prime}$ be the longest dyadic interval containing $I$ so that

$$
I^{\prime} \times J \subseteq \Omega^{*}=\left\{x \in \mathbb{R}^{2 n} \mid \mathcal{M}_{s}\left(\chi_{\Omega}\right)(x)>1 / 2\right\} .
$$

Then $I^{\prime} \times J$ is in $m_{1}\left(\Omega^{*}\right)$. Let $S$ be the longest dyadic interval so that $S \supseteq J$ and $I^{\prime} \times S \subseteq \Omega^{* *}$ where

$$
\Omega^{* *}=\left\{x \in \mathbb{R}^{2 n} \mid \mathcal{M}_{s}\left(\chi_{\Omega^{*}}\right)(x)>1 / 2\right\} .
$$

Let $\widetilde{R}$ be the 10 -fold dilate of $I^{\prime} \times S$ concentric with $I^{\prime} \times S$. Clearly, an application of the strong maximal theorem (see $[8,19]$ for the proof) shows that

$$
|\bigcup \widetilde{R}| \leq c\left|\Omega^{* *}\right| \leq c\left|\Omega^{*}\right| \leq c|\Omega| \text {. }
$$

We then have

$$
\begin{aligned}
\int_{\bigcup \widetilde{R}} a_{L}^{*}(x) d x & \leq C|\bigcup \widetilde{R}|^{1 / 2}\left\|a_{L}^{*}\right\|_{L^{2}\left(\mathbb{R}^{2 n}\right)} \leq C|\bigcup \widetilde{R}|^{1 / 2}\left\|\mathcal{M}_{S}(a)\right\|_{L^{2}\left(\mathbb{R}^{2 n}\right)} \\
& \leq C|\Omega|^{1 / 2}\|a\|_{L^{2}\left(\mathbb{R}^{2 n}\right)} \leq C|\Omega|^{1 / 2}|\Omega|^{-1 / 2} \leq C .
\end{aligned}
$$

We now find an estimate for

$$
\int_{(\cup \widetilde{R})^{c}} a_{L}^{*}(x) d x \leq C .
$$

We can write

$$
\begin{aligned}
\int_{(\cup \widetilde{R})^{c}} a_{L}^{*}(x) d x & \leq \sum_{R \in m(\Omega)} \int_{(\widetilde{R})^{c}}\left(a_{R}\right)_{L}^{*}(x) d x \\
& \leq \sum_{R \in m(\Omega)} \int_{x_{1} \notin 10 I^{\prime}}\left(a_{R}\right)_{L}^{*}(x) d x+\sum_{R \in m(\Omega)} \int_{x_{2} \notin 10 S}\left(a_{R}\right)_{L}^{*}(x) d x .
\end{aligned}
$$

We only need to calculate the estimate for the first term above since the proof of the estimate for the second term is similar.

Observe that

$$
\begin{aligned}
\sum_{R \in m(\Omega)} \int_{x_{1} \notin 10 I^{\prime}}\left(a_{R}\right)_{L}^{*}(x) d x & =\sum_{R \in m(\Omega)}\left(\int_{x_{1} \notin 10 I^{\prime}} \int_{x_{2} \in 10 J}+\int_{x_{1} \notin 10 I^{\prime}} \int_{x_{2} \notin 10 J}\right)\left(a_{R}\right)_{L}^{*}(x) d x \\
& =: \mathrm{E}_{1}+\mathrm{E}_{2} .
\end{aligned}
$$


By Hölder's inequality, we have

$$
\begin{aligned}
& \mathrm{E}_{1} \leq \sum_{R \in m(\Omega)}|J|^{1 / 2} \int_{x_{1} \notin 100 I^{\prime}}\left\|\left(a_{R}\right)_{L}^{*}\left(x_{1}, \cdot\right)\right\|_{L^{2}\left(d x_{2}\right)} d x_{1} \\
& \leq C \sum_{R \in m(\Omega)}|J|^{1 / 2} \int_{x_{1} \notin 100 I^{\prime}}\left\{\int_{\mathbb{R}^{n}} \sup _{\left|x_{1}-y_{1}\right|<t_{1}}\left|e^{-t_{1} \sqrt{L}} a_{R}\left(y_{1}, x_{2}\right)\right|^{2} d x_{2}\right\}^{1 / 2} d x_{1}
\end{aligned}
$$

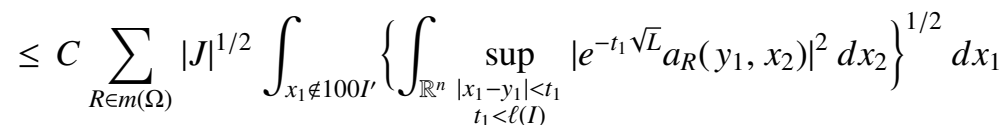

$$
\begin{aligned}
& +C \sum_{R \in m(\Omega)}|J|^{1 / 2} \int_{x_{1} \notin 100 I^{\prime}}\left\{\int_{\mathbb{R}^{n}} \sup _{\substack{\left|x_{1}-y_{1}\right|<t_{1} \\
t_{1} \geq \ell(I)}}\left|e^{-t_{1} \sqrt{L}} a_{R}\left(y_{1}, x_{2}\right)\right|^{2} d x_{2}\right\}^{1 / 2} d x_{1} \\
& =: \mathrm{E}_{11}+\mathrm{E}_{12} \text {. }
\end{aligned}
$$

We consider the term $\mathrm{E}_{11}$ above. Let $x_{I}$ denote the center of cube $I$. Note that $x_{1} \notin 100 I^{\prime}$ and $\left|x_{1}-y_{1}\right|<t_{1}<\ell(I)$. It follows from the estimate (4.2) that

$$
\begin{aligned}
\left|e^{-t_{1} \sqrt{L}} a_{R}\left(\cdot, x_{2}\right)\left(y_{1}\right)\right| & \leq C \int_{\mathbb{R}^{n}} \frac{t_{1}}{\left(t_{1}+\left|y_{1}-z_{1}\right|\right)^{n+1}}\left|a_{R}\left(z_{1}, x_{2}\right)\right| d z_{1} \\
& \leq C \frac{\ell(I)}{\left|x_{1}-x_{I}\right|^{n+1}}\left\|a_{R}\left(\cdot, x_{2}\right)\right\|_{L^{1}\left(\mathbb{R}^{n}\right)} \\
& \leq C|I|^{1 / 2} \frac{\ell(I)}{\left|x_{1}-x_{I}\right|^{n+1}}\left\|a_{R}\left(\cdot, x_{2}\right)\right\|_{L^{2}\left(\mathbb{R}^{n}\right)}
\end{aligned}
$$

which, in combination with Lemma 4.1, gives us that

$$
\begin{aligned}
\mathrm{E}_{11} & \leq C \sum_{R \in m(\Omega)}|J|^{1 / 2}|I|^{1 / 2}\left\{\int_{x_{1} \notin 100 I^{\prime}} \frac{\ell(I)}{\left|x_{1}-x_{I}\right|^{n+1}} d x_{1}\right\}\left\|a_{R}\right\|_{L^{2}\left(\mathbb{R}^{2 n}\right)} \\
& \leq C \sum_{R \in m(\Omega)}|R|^{1 / 2}\left\|a_{R}\right\|_{L^{2}\left(\mathbb{R}^{2 n}\right)} \gamma_{1}(R)^{-1} \\
& \leq C\left(\sum_{R \in m(\Omega)}\left\|a_{R}\right\|_{L^{2}\left(\mathbb{R}^{2 n}\right)}^{2}\right)^{1 / 2}\left(\sum_{R \in m(\Omega)}|R| \gamma_{1}(R)^{-2}\right)^{1 / 2} \\
& \leq C .
\end{aligned}
$$

For the term $\mathrm{E}_{12}$ above, we apply the definition of the product $(1,2)$-atom to obtain

$$
\begin{aligned}
\mid e^{-t_{1} \sqrt{L}} & a_{R}\left(\cdot, x_{2}\right)\left(y_{1}\right) \mid \\
& \leq\left(\frac{\ell(I)}{t_{1}}\right)^{2}\left|t_{1}^{2} L e^{-t_{1} \sqrt{L}} \ell(I)^{-2}\left(L^{0} \otimes L^{1}\right) b_{R}\left(\cdot, x_{2}\right)\left(y_{1}\right)\right| \\
& \leq C\left(\frac{\ell(I)}{t_{1}}\right)^{2} \int_{\mathbb{R}^{n}} \frac{t_{1}}{\left(t_{1}+\left|y_{1}-z_{1}\right|\right)^{n+1}}\left|\ell(I)^{-2}\left(L^{0} \otimes L^{1}\right) b_{R}\left(\cdot, x_{2}\right)\left(z_{1}\right)\right| d z_{1} .
\end{aligned}
$$


Note that

$$
x_{1} \notin 100 I^{\prime},\left|x_{1}-y_{1}\right|<t_{1}, \ell(I) \leq t_{1}, z_{1} \in I .
$$

We can obtain the estimate

$$
t_{1}+\left|y_{1}-z_{1}\right| \geq\left|x_{1}-x_{I}\right| / 2
$$

and deduce that

$$
\left|e^{-t_{1} \sqrt{L}} a_{R}\left(\cdot, x_{2}\right)\left(y_{1}\right)\right| \leq C \frac{\ell(I)}{\left|x_{1}-x_{I}\right|^{n+1}}\left\|\ell(I)^{-2}\left(L^{0} \otimes L^{1}\right) b_{R}\left(\cdot, x_{2}\right)\right\|_{L^{1}\left(\mathbb{R}^{n}\right)} .
$$

It follows from (4.4) and Hölder's inequality that

$$
\begin{aligned}
\mathrm{E}_{12} \leq & C \sum_{R \in m(\Omega)}|J|^{1 / 2}|I|^{1 / 2} \int_{x_{1} \notin 100 I^{\prime}} \frac{\ell(I)}{\left|x_{1}-x_{I}\right|^{n+1}} d x_{1} \\
& \times\left\|\ell(I)^{-2}\left(L^{0} \otimes L^{1}\right) b_{R}\right\|_{L^{2}\left(\mathbb{R}^{2 n}\right)} \\
\leq & C \sum_{R \in m(\Omega)}|R|^{1 / 2} \gamma_{1}(R)^{-1}\left\|\ell(I)^{-2}\left(L^{0} \otimes L^{1}\right) b_{R}\right\|_{L^{2}\left(\mathbb{R}^{2 n}\right)} \\
\leq & C\left(\sum_{R \in m(\Omega)}|R| \gamma_{1}(R)^{-2}\right)^{1 / 2}\left(\sum_{R \in m(\Omega)} \ell(I)^{-4}\left\|\left(L^{0} \otimes L^{1}\right) b_{R}\right\|_{L^{2}\left(\mathbb{R}^{2 n}\right)}^{2}\right) \\
\leq & C
\end{aligned}
$$

which, together with the estimate of $\mathrm{E}_{11}$, gives us that $\mathrm{E}_{1} \leq C$.

Consider the term $\mathrm{E}_{2}$. We first estimate the maximal function $\left(a_{R}\right)_{L}^{*}$. Now

$$
\begin{aligned}
\left(a_{R}\right)_{L}^{*}(x)= & \sup _{\left|y_{2}-x_{2}\right|<t_{2}} \sup _{\left|y_{1}-x_{1}\right|<t_{1}}\left|e^{-t_{1} \sqrt{L}} \otimes e^{-t_{2} \sqrt{L}} a_{R}\left(y_{1}, y_{2}\right)\right| \\
\leq & \sup _{\substack{y_{2}-x_{2} \mid<t_{2} \\
t_{2}<\ell(J)}} \sup _{\substack{\left|y_{1}-x_{1}\right|<t_{1} \\
t_{1}<\ell(I)}}\left|e^{-t_{1} \sqrt{L}} \otimes e^{-t_{2} \sqrt{L}} a_{R}\left(y_{1}, y_{2}\right)\right| \\
& +\sup _{\substack{\left|y_{2}-x_{2}\right|<t_{2} \\
t_{2}<\ell(J)}} \sup _{\substack{\left|y_{1}-x_{1}\right|<t_{1} \\
t_{1} \geq \ell(I)}}\left|e^{-t_{1} \sqrt{L}} \otimes e^{-t_{2} \sqrt{L}} a_{R}\left(y_{1}, y_{2}\right)\right| \\
& +\sup _{\substack{\left|y_{2}-x_{2}\right|<t_{2}\left|y_{1}-x_{1}\right|<t_{1} \\
t_{2} \geq \ell(J)}}\left|e^{-t_{1} \sqrt{L}} \otimes e^{-t_{2} \sqrt{L}} a_{R}\left(y_{1}, y_{2}\right)\right| \\
& +\sup _{\substack{\left|y_{2}-x_{2}\right|<t_{2}\left|y_{1}-x_{1}\right|<t_{1} \\
t_{2} \geq \ell(J)}}\left|e^{-t_{1} \sqrt{L}} \otimes e^{-t_{2} \sqrt{L}} a_{R}\left(y_{1}, y_{2}\right)\right| \\
=: & \mathrm{E}_{21}+\mathrm{E}_{22}+\mathrm{E}_{23}+\mathrm{E}_{24} .
\end{aligned}
$$

We only need to estimate the term $\mathrm{E}_{22}$ since the estimates of the remaining terms are similar. 
Applying (4.4) with $a_{R}\left(\cdot, x_{2}\right)$ replaced by $e^{-t_{2} \sqrt{L}} a_{R}\left(\cdot, y_{2}\right)$, we obtain

$$
\begin{aligned}
\mathrm{E}_{22} & \leq C \sup _{\substack{\left|y_{2}-x_{2}\right|<t_{2} \\
t_{2}<\ell(J)}} \frac{\ell(I)}{\left|x_{1}-x_{I}\right|^{n+1}}\left\|\ell(I)^{-2}\left(L^{0} \otimes e^{-t_{2} \sqrt{L}} L^{1}\right) b_{R}\left(\cdot, y_{2}\right)\right\|_{L^{1}\left(\mathbb{R}^{n}\right)} \\
& \leq C \frac{\ell(I)}{\left|x_{1}-x_{I}\right|^{n+1}}\left\|\sup _{\substack{\left|y_{2}-x_{2}\right|<t_{2} \\
t_{2}<\ell(J)}}\left|\left(\ell(I)^{-2} L^{0} \otimes e^{-t_{2} \sqrt{L}} L^{1}\right) b_{R}\left(\cdot, y_{2}\right)\right|\right\|_{L^{1}\left(\mathbb{R}^{n}\right)} .
\end{aligned}
$$

Applying (4.3) with $a_{R}\left(\cdot, x_{2}\right)$ replaced by $\left(\ell(I)^{-2} L^{0} \otimes L^{1}\right) b_{R}\left(x_{1}, \cdot\right)$, together with Hölder's inequality, we obtain

$$
\begin{aligned}
\mathrm{E}_{22} & \leq C \frac{\ell(I)}{\left|x_{1}-x_{I}\right|^{n+1}} \frac{\ell(J)}{\left|x_{2}-x_{J}\right|^{n+1}}\left\|\left(\ell(I)^{-2} L^{0} \otimes L^{1}\right) b_{R}\right\|_{L^{1}\left(\mathbb{R}^{n} \times \mathbb{R}^{n}\right)} \\
& \leq C \frac{\ell(I)}{\left|x_{1}-x_{I}\right|^{n+1}} \frac{\ell(J)}{\left|x_{2}-x_{J}\right|^{n+1}}|R|^{1 / 2}\left\|\left(\ell(I)^{-2} L^{0} \otimes L^{1}\right) b_{R}\right\|_{L^{2}\left(\mathbb{R}^{n} \times \mathbb{R}^{n}\right)} .
\end{aligned}
$$

A similar argument to that given for $\mathrm{E}_{22}$ shows that

$$
\begin{aligned}
\left(a_{R}\right)_{L}^{*}(x) \leq C & \frac{\ell(J)}{\left|x_{2}-x_{J}\right|^{n+1}} \frac{\ell(I)}{\left|x_{1}-x_{I}\right|^{n+1}}|R|^{1 / 2} \\
& \times \sum_{i, j=0}^{1} \ell(I)^{2 i-2} \ell(J)^{2 j-2}\left\|\left(L^{i} \otimes L^{j}\right) b_{R}\right\|_{L^{2}\left(\mathbb{R}^{n} \times \mathbb{R}^{n}\right)} .
\end{aligned}
$$

Hence

$$
\begin{aligned}
\mathrm{E}_{2} & =\sum_{R \in m(\Omega)} \int_{x_{1} \notin 10 I^{\prime}} \int_{x_{2} \notin 10 J}\left(a_{R}\right)_{L}^{*}(x) d x \\
& \leq \sum_{R \in m(\Omega)} \ell(I) / \ell\left(I^{\prime}\right)|R|^{1 / 2} \sum_{i, j=0}^{1} \ell(I)^{2 i-2} \ell(J)^{2 j-2}\left\|\left(L^{i} \otimes L^{j}\right) b_{R}\right\|_{L^{2}\left(\mathbb{R}^{n} \times \mathbb{R}^{n}\right)} \\
& \leq C \sum_{R \in m(\Omega)}|R|^{1 / 2} \gamma_{1}(R)^{-1} \sum_{i, j=0}^{1} \ell(I)^{2 i-2} \ell(J)^{2 j-2}\left\|\left(L^{i} \otimes L^{j}\right) b_{R}\right\|_{L^{2}\left(\mathbb{R}^{n} \times \mathbb{R}^{n}\right)}
\end{aligned}
$$

Applying Lemma 4.1 and the definition of product $(1,2)$-atom, together with Hölder's inequality, we obtain the estimate $\mathrm{E}_{2} \leq C$. We thus obtain the required estimate $\left\|a_{L}^{*}\right\|_{L^{1}\left(\mathbb{R}^{2 n}\right)} \leq C$ and can deduce that $f \in H_{L}^{1}\left(\mathbb{R}^{n} \times \mathbb{R}^{n}\right)$.

Step 2. Let

$$
f \in H_{L}^{1}\left(\mathbb{R}^{n} \times \mathbb{R}^{n}\right) \cap L^{2}\left(\mathbb{R}^{n} \times \mathbb{R}^{n}\right)
$$

We begin with a version of the Calderón reproducing formula. Let $\Psi(x)=x^{4} \Phi(x)$ be the function in Lemma 2.1. Since $f \in L^{2}\left(\mathbb{R}^{n} \times \mathbb{R}^{n}\right)$, applying the $L^{2}$-functional calculus gives us that

$$
f=c_{\Psi} \int_{0}^{\infty} \int_{0}^{\infty} \Psi_{t_{1}}(\sqrt{L}) t_{1} \sqrt{L} e^{-t_{1} \sqrt{L}} \otimes \Psi_{t_{2}}(\sqrt{L}) t_{2} \sqrt{L} e^{-t_{2} \sqrt{L}} f \frac{d t}{t_{1} t_{2}} .
$$


For $k=0, \pm 1, \ldots$ we set

$$
\begin{gathered}
E_{k}=\left\{x \mid f_{L}^{*}(x)>2^{k}\right\}, \\
E_{k}^{*}=\left\{x \mid \mathcal{M}_{s} \chi_{E_{k}}(x)>2^{-(2 n+1)}\right\}
\end{gathered}
$$

and

$$
E_{k}^{* *}=\left\{x \mid \mathcal{M}_{s} \chi_{E_{k}^{*}}(x)>(4 n)^{-n}\right\}
$$

Then

$$
E_{k} \subseteq E_{k}^{*} \subseteq E_{k}^{* *} \quad \text { and } \quad\left|E_{k}^{* *}\right| \leq C\left|E_{k}^{*}\right| \leq C^{\prime}\left|E_{k}\right| .
$$

We define $T_{k}:=\widehat{E_{k}^{*}} \backslash \widehat{E_{k+1}^{*}}$ and apply formula (4.5) to write

$$
f=\sum_{k \in \mathbb{Z}} \lambda_{k} a_{k}
$$

where $\lambda_{k}=2^{k}\left|E_{k}^{*}\right|$ and

$$
a_{k}=\lambda_{k}^{-1} c \Psi \int_{0}^{\infty} \int_{0}^{\infty} \Psi_{t_{1}}(\sqrt{L}) \Psi_{t_{2}}(\sqrt{L})\left(\chi_{T_{k}} t_{1} \sqrt{L} e^{-t_{1} \sqrt{L}} \otimes t_{2} \sqrt{L} e^{-t_{2} \sqrt{L}}\right) f \frac{d t}{t_{1} t_{2}} .
$$

It is clear that

$$
\sum_{k}\left|\lambda_{k}\right| \leq C \sum_{k} 2^{k}\left|E_{k}^{*}\right| \leq C\left\|f_{L}^{*}\right\|_{L^{1}\left(\mathbb{R}^{2 n}\right)}
$$

We claim that for each $k \in \mathbb{Z}$ the term $a_{k}$ is a product $(1,2)$-atom associated with the open set $E_{k}^{* *}$ for some constant $C$.

Let us prove the claim. First, it follows by Lemma 2.1 that the integral kernel $K_{\Psi_{t}(\sqrt{L})}$ of the operator $\Psi_{t}(\sqrt{L})$ has its support contained in

$$
\left\{(x, y) \in \mathbb{R}^{n} \times \mathbb{R}^{n}|| x_{1}-y_{1}\left|\leq t_{1},\right| x_{2}-y_{2} \mid \leq t_{2}\right\} .
$$

This, together with the definition of $T_{k}$, shows that supp $a_{k} \subseteq E_{k}^{* *}$. Second, for any dyadic rectangle $R=I \times J$ of $\mathbb{R}^{n} \times \mathbb{R}^{n}$, we define

$$
R^{+}=\left\{\left(y_{1}, y_{2}, t_{1}, t_{2}\right) \mid y_{1} \in I, y_{2} \in J, \frac{\ell(I)}{2}<t_{1} \leq \ell(I), \frac{\ell(J)}{2}<t_{1} \leq \ell(J)\right\} .
$$

It can be verified that if $T_{k} \cap R^{+} \neq \varnothing$, then $R \subseteq E_{k}^{* *}$. Applying the definition of $R^{+}$, we obtain $T_{k}=\bigcup_{R}\left(T_{k} \cap R^{+}\right)$where the $R$ are all dyadic rectangles of $\mathbb{R}^{n} \times \mathbb{R}^{n}$. We can further decompose $a_{k}$ as follows:

$$
\begin{aligned}
a_{k}= & \sum_{\bar{R} \in m\left(E_{k}^{* *}\right)} \sum_{R \subseteq \bar{R}} \lambda_{k}^{-1} \\
& \times \int_{0}^{\infty} \int_{0}^{\infty} \Psi_{t_{1}}(\sqrt{L}) \Psi_{t_{2}}(\sqrt{L})\left(\chi_{T_{k}} t_{1} \sqrt{L} e^{-t_{1} \sqrt{L}} \otimes t_{2} \sqrt{L} e^{-t_{2} \sqrt{L}}\right) f \frac{d t}{t_{1} t_{2}} \\
= & \sum_{\bar{R} \in m\left(E_{k}^{* *}\right)} a_{k, \bar{R}}=: \sum_{\bar{R} \in m\left(E_{k}^{* *}\right)}(L \otimes L) b_{k, \bar{R}}
\end{aligned}
$$

where $m\left(E_{k}^{* *}\right)$ denotes the set of all maximal dyadic rectangles of $E_{k}^{* *}$. 
By Lemma 2.1, if $i, j=0,1$, then

$$
\operatorname{supp}\left(\left(L^{i} \otimes L^{j}\right) b_{k, \bar{R}}\right) \subseteq 2 \bar{R} .
$$

To continue, for each $\bar{R}$ we consider some $h \in L^{2}(\bar{R})$ such that $\|h\|_{L^{2}(\bar{R})}=1$. Then for every $k \in \mathbb{Z}$, we have

$$
\begin{aligned}
\left\|a_{k, \bar{R}}\right\|_{L^{2}} & =\sup _{\|h\|_{L^{2}} \leq 1}\left|\left\langle a_{k, \bar{R}}, h\right\rangle\right| \\
& \leq C 2^{-k}\left|E_{k}^{*}\right|^{-1} \times\left(\sum_{R \subseteq \bar{R}} \int_{T_{k} \cap R^{+}}\left|t_{1} \nabla_{X_{1}} e^{-t_{1} \sqrt{L}} \otimes t_{2} \nabla_{X_{2}} e^{-t_{2} \sqrt{L}} f\left(y_{1}, y_{2}\right)\right|^{2} \frac{d y d t}{t_{1} t_{2}}\right)^{1 / 2} .
\end{aligned}
$$

In order to verify that

$$
\sum_{\bar{R} \in m\left(E_{k}^{* *}\right)}\left\|a_{k, \bar{R}}\right\|_{2}^{2} \leq C\left|E_{k}^{* *}\right|^{-1}
$$

it is enough to prove that

$$
\int_{T_{k}}\left|t_{1} \nabla_{X_{1}} e^{-t_{1} \sqrt{L}} \otimes t_{2} \nabla_{X_{2}} e^{-t_{2} \sqrt{L}} f\left(y_{1}, y_{2}\right)\right|^{2} \frac{d y d t}{t_{1} t_{2}} \leq C 2^{2 k}\left|E_{k}^{*}\right| .
$$

We now prove inequality (4.6). Let $\varphi \in C_{0}^{\infty}$ be the function in Lemma 4.2 and set $F_{k}=E_{k}^{*} \backslash E_{k+1}$. The argument given to prove formula (3.3) shows that

$$
\left|\left(\varphi_{t_{1}} \otimes \varphi_{t_{2}}\right) * \chi_{F}(y)\right| \geq C
$$

for all $(y, t) \in T_{k}$. This, together with Lemma 4.2, gives us that

$$
\begin{aligned}
& \int_{T_{k}}\left|t_{1} \nabla_{X_{1}} e^{-t_{1} \sqrt{L}} \otimes t_{2} \nabla_{X_{2}} e^{-t_{2} \sqrt{L}} f\left(y_{1}, y_{2}\right)\right|^{2} \frac{d y d t}{t_{1} t_{2}} \\
& \leq \int_{\mathbb{R}_{+}^{n+1} \times \mathbb{R}_{+}^{n+1}}\left|t_{1} \nabla_{X_{1}} e^{-t_{1} \sqrt{L}} \otimes t_{2} \nabla_{X_{2}} e^{-t_{2} \sqrt{L}} f\left(y_{1}, y_{2}\right)\right|^{2}\left|\left(\varphi_{t_{1}} \otimes \varphi_{t_{2}}\right) * \chi_{F_{k}}(y)\right|^{2} \frac{d y d t}{t_{1} t_{2}} \\
& \leq \int_{\mathbb{R}_{+}^{n+1} \times \mathbb{R}_{+}^{n+1}}\left|e^{-t_{1} \sqrt{L}} \otimes e^{-t_{2} \sqrt{L}} f\left(y_{1}, y_{2}\right)\right|^{2}\left|\left(\psi_{t_{1}}^{(1)} \otimes \psi_{t_{2}}^{(2)}\right) * \chi_{F_{k}}\left(y_{1}, y_{2}\right)\right|^{2} \frac{d y d t}{t_{1} t_{2}} \\
&+\int_{\mathbb{R}_{+}^{n+1} \times \mathbb{R}^{n}}\left|e^{-t_{1} \sqrt{L}} f\left(y_{1}, x_{2}\right)\right|^{2}\left|\psi_{t_{1}}^{(1)} * \chi_{F_{k}}\left(y_{1}, x_{2}\right)\right|^{2} \frac{d y_{1} d t_{1}}{t_{1}} d x_{2} \\
&+\int_{\mathbb{R}^{n} \times \mathbb{R}_{+}^{n+1}}\left|e^{-t_{2} \sqrt{L}} f\left(x_{1}, y_{2}\right)\right|^{2}\left|\psi_{t_{2}}^{(2)} * \chi_{F_{k}}\left(x_{1}, y_{2}\right)\right|^{2} \frac{d y_{2} d t_{2}}{t_{2}} d x_{1} \\
&+\int_{\mathbb{R}^{n} \times \mathbb{R}^{n}}\left|f\left(x_{1}, x_{2}\right)\right|^{2}\left|\chi_{F_{k}}\left(x_{1}, x_{2}\right)\right|^{2} d x_{1} d x_{2} \\
&=: \mathrm{I}_{1}+\mathrm{I}_{2}+\mathrm{I}_{3}+\mathrm{I}_{4} .
\end{aligned}
$$

In order to estimate the term $\mathrm{I}_{1}$, we note that if

$$
\left(\psi_{t_{1}}^{(1)} \otimes \psi_{t_{2}}^{(2)}\right) * \chi_{F_{k}}\left(y_{1}, y_{2}\right) \neq 0
$$


then $F_{k} \cap B_{y, t} \neq \varnothing$. Moreover, there exists

$$
x^{0}=\left(x_{1}^{0}, x_{2}^{0}\right) \in B_{y, t} \cap E_{k}^{*} \cap{ }^{c} E_{k+1}
$$

and we have

$$
\left|e^{-t_{1} \sqrt{L}} \otimes e^{-t_{2} \sqrt{L}} f\left(y_{1}, y_{2}\right)\right| \leq f_{L}^{*}\left(x^{0}\right) \leq 2^{k+1}
$$

which gives us that $\mathrm{I}_{1} \leq C 2^{2 k}\left|E_{k}^{*}\right|$. We similarly have

$$
\mathrm{I}_{2}+\mathrm{I}_{3} \leq C 2^{2 k}\left|E_{k}^{*}\right| \text {. }
$$

We now obtain an estimate for the term $\mathrm{I}_{4}$. It follows from the inequality $f\left(x_{1}, x_{2}\right) \leq$ $f_{L}^{*}\left(x_{1}, x_{2}\right)$ that

$$
\mathrm{I}_{4} \leq C 2^{2 k}\left|E_{k}^{*}\right|
$$

The desired estimate (4.6) follows easily and then

$$
\sum_{\bar{R} \in m\left(E_{k}^{* *}\right)}\left\|a_{k, \bar{R}}\right\|_{L^{2}\left(\mathbb{R}^{2 n}\right)}^{2} \leq C\left|E_{k}^{* *}\right|^{-1}
$$

A similar argument to the one given above shows that

$$
\sum_{\bar{R} \in m\left(E_{k}^{* *}\right)} \sum_{i, j=0}^{1} \ell(I)^{4 i-4} \ell(J)^{4 j-4}\left\|\left(L^{i} \otimes L^{j}\right) b_{k, \bar{R}}\right\|_{L^{2}\left(\mathbb{R}^{2 n}\right)}^{2} \leq C\left|E_{k}^{* *}\right|^{-1} .
$$

We have shown that for every $k \in \mathbb{Z}$ the expression $C^{-1} a_{k}$ is a product $(1,2)$-atom associated with the open set $E_{k}^{* *}$ for some constant $C$. This shows that $f$ has an decomposition of the form given in (1.3). The proof of Theorem 1.2 is complete.

\section{Acknowledgements}

The authors would like to express their gratitude to the referees for their careful reading of the manuscript, and for offering numerous valuable suggestions to improve its mathematical accuracy.

\section{References}

[1] P. Auscher, A. McIntosh and E. Russ, 'Hardy spaces of differential forms on Riemannian manifolds', J. Geom. Anal. 18 (2008), 192-248.

[2] P. Auscher and E. Russ, 'Hardy spaces and divergence operators on strongly Lipschitz domain of $\mathbb{R}^{n}$, J. Funct. Anal. 201 (2003), 148-184.

[3] F. Bernicot and J. Zhao, 'New abstract Hardy spaces', J. Funct. Anal. 255 (2008), 1761-1796.

[4] S.-Y. A. Chang and R. Fefferman, 'A continuous version of duality of $H^{1}$ with BMO on the bidisc', Ann. of Math. (2) 112 (1980), 179-201.

[5] S.-Y. A. Chang and R. Fefferman, 'The Calderón-Zygmund decomposition on product domains', Amer. J. Math. 104 (1982), 445-468.

[6] J. Cheeger, M. Gromov and M. Taylor, 'Finite propagation speed, kernel estimates for functions of the Laplace operator, and the geometry of complete Riemannian manifolds', J. Differential Geom. 17 (1982), 15-53. 
[7] R. Coifman, 'A real-variable characterization of $H^{p}$ ', Studia Math. 51 (1974), 269-274.

[8] A. Córdoba and R. Fefferman, 'A geometric proof of the strong maximal theorem', Ann. of Math. (2) 102 (1975), 95-100.

[9] T. Coulhon and A. Sikora, 'Gaussian heat kernel upper bounds via Phragmén-Lindelöf theorem', Proc. Lond. Math. Soc. 96 (2008), 507-544.

[10] E. B. Davies, Heat Kernels and Spectral Theory (Cambridge University Press, Cambridge, 1989).

[11] D. G. Deng, L. Song, C. Q. Tan and L. X. Yan, 'Duality of Hardy and BMO spaces associated with operators with heat kernel bounds on product domains', J. Geom. Anal. 17 (2007), 455-483.

[12] X. T. Duong and L. X. Yan, 'Duality of Hardy and BMO spaces associated with operators with heat kernel bounds', J. Amer. Math. Soc. 18 (2005), 943-973.

[13] J. Dziubański and J. Zienkiewicz, 'Hardy spaces $H^{1}$ associated with Schrödinger operator with potential satisfying reverse Hölder inequality', Rev. Mat. Iberoam. 15 (1999), 279-296.

[14] R. Fefferman, 'Calderón-Zygmund theory for product domains: $H^{p}$ spaces', Proc. Natl. Acad. Sci. USA 83 (1986), 840-843.

[15] C. Fefferman and E. M. Stein, ' $H^{p}$ spaces of several variables', Acta Math. 129 (1972), 137-193.

[16] D. Goldberg, 'A local version of real Hardy spaces', Duke Math. J. 46 (1979), $27-42$.

[17] S. Hofmann, G. Z. Lu, D. Mitrea, M. Mitrea and L. X. Yan, 'Hardy spaces associated to nonnegative self-adjoint operators satisfying Davies-Gaffney estimates', Mem. Amer. Math. Soc., to appear.

[18] S. Hofmann and S. Mayboroda, 'Hardy and BMO spaces associated to divergence form elliptic operators', Math. Ann. 344 (2009), 37-116.

[19] B. Jessen, J. Marcinkiewicz and A. Zygmund, 'Note on the differentiability of multiple integrals', Fund. Math. 25 (1935), 217-234.

[20] J. L. Journé, ‘A covering lemma for product spaces', Proc. Amer. Math. Soc. 96 (1986), 593-598.

[21] R. H. Latter, 'A characterization of $H^{p}\left(\mathbb{R}^{n}\right)$ in terms of atoms', Studia Math. 62 (1978), 93-101.

[22] A. McIntosh, 'Operators which have an $H_{\infty}$ functional calculus', in: Miniconference on Operator Theory and Partial Differential Equations, 1986, Proceedings of the Centre for Mathematical Analysis, 14 (Australian National University, Canberra, 1986), pp. 210-231.

[23] K. Merryfield, 'On the area integral, Carleson measures and $H^{p}$ in the polydisc', Indiana Univ. Math. J. 34 (1985), 663-685.

[24] E. M. Ouhabaz, Analysis of Heat Equations on Domains, London Mathematical Society Monographs Series, 31 (Princeton University Press, Princeton, NJ, 2005).

[25] B. Simon, 'Maximal and minimal Schrödinger forms', J. Operator Theory 1 (1979), 37-47.

[26] E. M. Stein and G. Weiss, 'On the theory of harmonic functions of several variables, I: the theory of $H^{p}$ spaces', Acta Math. 103 (1960), 25-62.

[27] J. M. Wilson, 'On the atomic decomposition for Hardy spaces', Pacific J. Math. 116 (1985), 201-207.

LIANG SONG, Department of Mathematics, Sun Yat-sen (Zhongshan) University, Guangzhou 510275, PR China

e-mail: songl@mail.sysu.edu.cn

CHAOQIANG TAN, Department of Mathematics, Shantou University, Shantou Guangdong 515063, PR China e-mail: cqtan@stu.edu.cn

\author{
LIXIN YAN, Department of Mathematics, Sun Yat-sen (Zhongshan) University, \\ Guangzhou 510275, PR China \\ e-mail: mcsylx@mail.sysu.edu.cn
}

